\title{
Intersubband-transition-induced phase matching
}

\author{
Gilad Almogy, Mordechai Segev, ${ }^{*}$ and Amnon Yariv \\ California Institute of Technology, Pasadena, California 91125
}

Received March 10, 1994

\begin{abstract}
We suggest the use of the refractive-index changes associated with the intersubband transitions in quantum wells for phase matching in nonlinear materials. An improvement in the conversion efficiency of mid-IR secondharmonic generation by almost 2 orders of magnitude over non-phase-matched bulk GaAs is predicted. We also show that the linear phase contributions of intersubband transitions used for resonant enhancement of secondharmonic generation must be considered, as they could limit the conversion efficiency by increasing the phase mismatch on one hand or offset the bulk's dispersion and lead to phase matching on the other.
\end{abstract}

The second-harmonic generation (SHG) efficiencies of nonlinear materials are often limited by their coherence lengths, ${ }^{1}$ and schemes for phase matching or quasi-phase matching ${ }^{2}$ need to be employed for reasonable conversion efficiencies to be achieved. Recently it was demonstrated that the refractive index of bulk material may be selectively altered by the large refractive-index contributions associated with the intersubband transitions (ISBT's) in quantum wells. ${ }^{3,4}$ In this Letter we suggest the use of these tailorable refractive-index contributions to offset the bulk's dispersion and obtain a phase-matched material at a selected wavelength. The phase matching obtained in this manner is intrinsic and requires no external process such as quasi-phase matching or angle tuning. We examine the contribution of this technique to the conversion efficiency of mid-IR radiation in GaAs phase matched by GaAs/AlGaAs n-doped quantum wells. Both the ideal case of a bulk material embedded with quantum wells and the case that is more practical as a result of growth limitations of the TM mode in a narrow dielectric waveguide ${ }^{5}$ are considered.

We consider ISBT index contributions originating either from symmetric (two-level) wells, designed for phase matching only, or from asymmetric (threelevel) quantum wells, designed both for resonant enhancement of the SHG and for phase matching. First we examine the former, in which the source of the SHG nonlinearity is the bulk material. Introduction into bulk material of a transition whose real part of the optical susceptibility will offset the bulk's dispersion will lead to phase matching. Doped quantum wells are optimal for this purpose, ${ }^{3}$ because the ISBT's constitute a two-level system with a designable energy-level separation and large optical dipole elements. In the following treatment we shall limit ourselves to electron ISBT's in quantum wells, but the treatment is valid for any two-level system that may be distributed uniformly (on a wavelength scale) in a dispersive host material.

The ISBT's offsetting the bulk's dispersion are unavoidably ${ }^{6}$ accompanied by loss; hence a solution of the SHG propagation equations that takes the absorption into account ${ }^{7}$ is necessary. Maxwell's equations for a field of $\omega$ and $2 \omega$ frequency components ( $E_{\omega}$ and $E_{2 \omega}$, respectively) reduce with the slowly varying amplitude approximation ${ }^{8}$ to

$$
\begin{aligned}
& \frac{\mathrm{d} E_{\omega}}{\mathrm{d} z}=-\alpha_{\omega} E_{\omega}-\frac{i \omega}{2 \varepsilon_{0} n_{\omega} c} d E_{2 \omega} E_{\omega}^{*} \exp (-i \Delta k z), \\
& \frac{\mathrm{d} E_{2 \omega}}{\mathrm{d} z}=-\alpha_{2 \omega} E_{2 \omega}-\frac{i \omega}{\varepsilon_{0} n_{2 \omega} c} d E_{\omega}^{2} \exp (i \Delta k z),
\end{aligned}
$$

where $n_{\omega}$ and $n_{2 \omega}$ are the first- and second-harmonic bulk refractive indices, respectively, $d$ is the bulk's SHG coefficient, and $\Delta k \equiv k_{2 \omega}-2 k_{\omega}$ is defined as the phase mismatch. The real and imaginary components of the ISBT linear susceptibility, $\chi^{\prime}(\omega)$ and $\chi^{\prime \prime}(\omega)$, are given for a two-level system as ${ }^{8}$

$$
\chi(\omega) \equiv \chi^{\prime}(\omega)-i \chi^{\prime \prime}(\omega)=\frac{N \mu^{2}}{\varepsilon_{0} \hbar} T_{2} \frac{\left(\Delta \omega T_{2}-i\right)}{\left(\Delta \omega T_{2}\right)^{2}+1},
$$

where $N$ is the average electron volume density, $\mu$ is the intersubband dipole element, $T_{2}$ is the dephasing time, and $\Delta \omega$ is the detuning of $\omega$ from the ISBT frequency $(\omega+\Delta \omega$ is hence the second harmonic's detuning). The wave vectors are thus modified by the real components of the ISBT and become

$$
k_{\omega}^{2}=\frac{\omega^{2}}{c^{2}}\left[n_{\omega}^{2}+\chi^{\prime}(\omega)\right],
$$

and the absorption, neglecting the bulk's contribution, is given by

$$
\alpha_{\omega}=\frac{\omega}{2 n_{\omega} c} \chi^{\prime \prime}(\omega)
$$

Normalizing the fundamental frequency and its detuning in half-linewidth units $\left(\tilde{\omega} \equiv \omega T_{2}\right.$ and $\Delta \tilde{\omega} \equiv$ $\left.\Delta \omega T_{2}\right)$, we obtain the phase-matching condition from Eqs. (1), (2), and (3a) as

$$
\begin{aligned}
\frac{n_{\omega}{ }^{2}-n_{2 \omega}{ }^{2}}{2 n_{\omega}} & \cong n_{\omega}-n_{2 \omega} \equiv \Delta n \\
& =\frac{\chi_{\mathrm{res}}}{2 n_{\omega}}\left[\frac{\Delta \tilde{\omega}}{\Delta \tilde{\omega}^{2}+1}-\frac{(\Delta \tilde{\omega}-\tilde{\omega})}{(\Delta \tilde{\omega}-\tilde{\omega})^{2}+1}\right],
\end{aligned}
$$

where $\chi_{\text {res }}$ is the on-resonance (imaginary) susceptibility $[\Delta \omega=0$ in Eq. (2)]. When phase matching $(\Delta k=0)$ is maintained, Eqs. (1) are solved as 


$$
\begin{aligned}
E_{2 \omega}(z)=- & i \frac{\omega}{\varepsilon_{0} n_{2 \omega} c} \frac{d}{\left(\alpha_{2 \omega}-2 \alpha_{\omega}\right)} \\
& \times\left[\exp \left(-2 \alpha_{\omega} z\right)-\exp \left(-\alpha_{2 \omega} z\right)\right] E_{\omega}(0)^{2},
\end{aligned}
$$

where we have neglected the depletion of the first harmonic that is due to SHG $\left(E_{2 \omega} \ll E_{\omega}\right)$. From Eq. (5) we find the optimal conversion distance as

$$
z_{\max }=\frac{1}{\alpha_{2 \omega}-2 \alpha_{\omega}} \ln \left(\frac{\alpha_{2 \omega}}{2 \alpha_{\omega}}\right)
$$

yielding a maximal (intensity) conversion efficiency of

$$
\begin{aligned}
& \eta\left(z_{\max }\right) \equiv \frac{I_{2 \omega}\left(z_{\max }\right)}{I_{\omega}(0)} \\
& =2\left(\frac{\mu_{0}}{\varepsilon_{0}}\right)^{3 / 2} \frac{\omega^{2} d^{2}}{n_{2 \omega} n_{\omega}^{2}}\left[\frac{1}{\alpha_{2 \omega}}\left(\frac{2 \alpha_{\omega}}{\alpha_{2 \omega}}\right)^{\frac{2 \alpha_{\omega}}{\alpha_{2 \omega}-2 \alpha_{\omega}}}\right]^{2} I_{\omega}(0),
\end{aligned}
$$

where $I_{\omega}(0)$ is the incident first-harmonic intensity. By substituting Eq. (2) and (3) and relation (4) into Eq. (6b), we find a maximum for the conversion efficiency at $\Delta \omega=\omega / 2$. Thus when the resonant transition is halfway between the fundamental and the second harmonic the conversion efficiency is given by

$$
\eta_{\max }=\frac{1}{2 e^{2}}\left(\frac{\mu_{0}}{\varepsilon_{0}}\right)^{3 / 2} \frac{d^{2} c^{2}}{n_{\omega}^{2} n_{2 \omega}} I_{\omega}\left(\frac{\tilde{\omega}}{\Delta n}\right)^{2},
$$

while the optimal propagation length for obtaining this maximal efficiency is

$$
z_{\max }=\frac{c T_{2}}{2 \Delta n}=\frac{\tilde{\omega}}{\pi} \frac{\lambda}{4 \Delta n}=\frac{\tilde{\omega}}{\pi} l_{c},
$$

where $\left(l_{c}=\lambda / 4 \Delta n\right)$ is the bulk's coherence length. ${ }^{8}$ The phase-matching condition becomes

$$
\Delta n=\frac{2}{n_{\omega}} \frac{N \mu^{2}}{\hbar \omega \varepsilon_{0}} \frac{2}{1+4 / \tilde{\omega}^{2}} \cong \frac{1}{n_{\omega}} \frac{N \mu^{2}}{\hbar \omega \varepsilon_{0}},
$$

and the conversion efficiency may thus be enhanced by a factor of as much as $(\tilde{\omega} / 2 e)^{2}$ over the nonphase-matched case. The enhancement is dependent only on the frequency-to-broadening ratio. For AlGaAs/GaAs quantum wells, $\tilde{\omega}$ 's of $\sim 25-50$ are typical, and the index mismatch is $\Delta n_{\mathrm{GaAs}} \cong 0.03$ (Ref. 9) for a first harmonic of $10.6 \mu \mathrm{m}$ doubled to $5.3 \mu \mathrm{m}$, leading to an improvement of almost 2 orders of magnitude over non-phase-matched bulk. To prevent saturation of the transitions, we must limit the incident optical intensity to $(\tilde{\omega} / 2)^{2}$ times the ISBT saturation intensity, ${ }^{7}$ but practically it will be limited by surface phenomena ${ }^{10}$ to $\sim 10 \mathrm{MW} / \mathrm{cm}^{2}$. This leads to a maximal conversion efficiency of approximately $3 \%$, with $d \cong 3.4 \times 10^{-21} \mathrm{~m} / \mathrm{V}$ for GaAs. ${ }^{11}$

The maximal refractive-index dispersion that may be compensated for is given by relation (7c) as a function of the average electron density and of the optical dipole matrix elements. AlGaAs/GaAs wells with a volume density of $10^{18} \mathrm{~cm}^{-3}$ and dipole matrix elements of $2 \mathrm{~nm}$ need a filling factor of less than $10 \%$ to correct the dispersion of bulk GaAs. Unfortunately current technology limits the growth of high-quality epitaxial layers to $\sim 10 \mu \mathrm{m}$, making waveguiding necessary for longer interaction distances. $^{5}$ The refractive-index contribution of ISBT's located in the core of a dielectric waveguide must be tailored to equalize the phase velocities of the first- and second-harmonic modes. Using a numerical solution ${ }^{12}$ of the TM modes in arbitrarily shaped dielectric waveguides, we find that phase matching should be obtainable by use of ISBT's, provided that sufficient confinement is achieved. Figure 1 shows a possible example of a phase-matched waveguide.

The refractive-index correction obtained by a single ISBT halfway between the first and second harmonics is insensitive to the transition's frequency to first order, making this phase-matching technique tolerant of quantum-well growth inaccuracies (ISBT frequency variations). The magnitude of the ISBT, however, has to be obtained with an accuracy of at least $\pi / \tilde{\omega}$ to increase the coherence length above the optimal propagation length. This magnitude is correctable by electron injection mechanisms, ${ }^{13}$ which are thus also the way tuning may be achieved. The phase matching is relatively broadband and can be tailored to cover most of the $\mathrm{CO}_{2}$ laser's spectral range, as shown in Fig. 2.

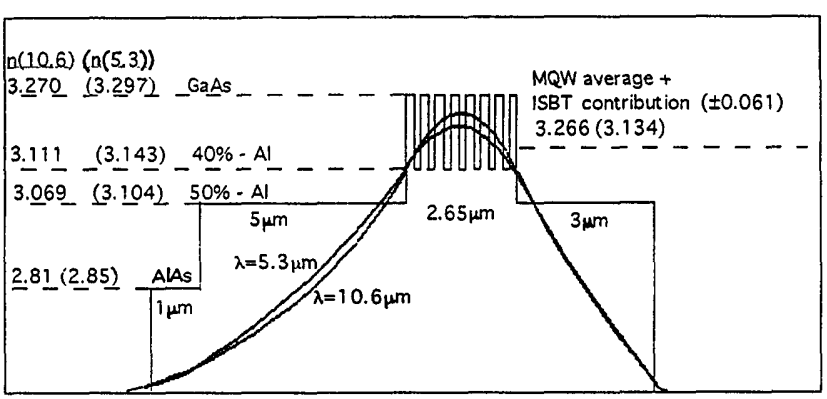

Fig. 1. Waveguide phase matched by the ISBT contributions to the susceptibility, consisting of the following layers: $\quad 1-\mu \mathrm{m}$ AlAs, $5-\mu \mathrm{m} \mathrm{Al} l_{0.5} \mathrm{GaAs}$ cladding, a multiple-quantum-well (MQW) stack of 150 periods of $6.2-\mathrm{nm}$ GaAs wells with $11.3-\mathrm{nm} \mathrm{Al}_{0.4} \mathrm{GaAs}$ barriers, n-doped to $6 \times 10^{11} \mathrm{~cm}^{-2}$ surface density, and a second, $3-\mu \mathrm{m}$ $\mathrm{Al}_{0.5} \mathrm{GaAs}$ cladding. The wave functions of the 10.6- and $5.3-\mu \mathrm{m}$ modes and the corresponding refractive indices are also shown.

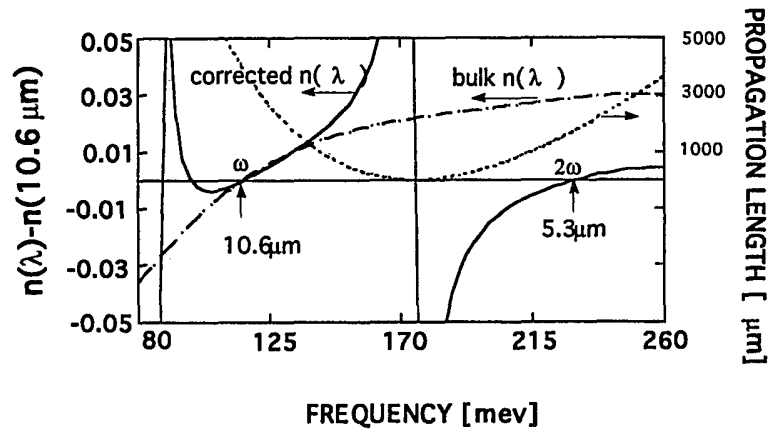

Fig. 2. Refractive-index (n) dispersion of GaAs: $n(\lambda)-n(10.6 \mu \mathrm{m})$ with a single correcting ISBT at $1.5 \omega$ is shown versus frequency (the solid curves). Also shown are the refractive-index dispersion of bulk GaAs (the dashed-dotted curve) and the propagation length, defined as the inverse of the energy-dependent ISBT-induced absorption coefficient (the dotted curve). 


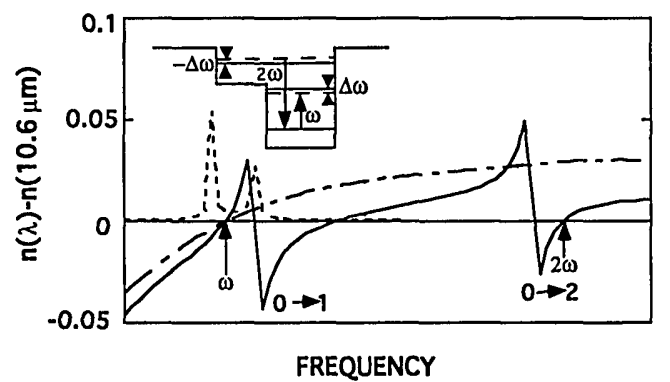

Fig. 3. Refractive-index (n) dispersion of GaAs: $n(\lambda)-n(10.6 \mu \mathrm{m})$ with two ISBT's used for resonant enhancement of the SHG as well as for phase matching shown versus frequency (solid curve). Also shown are the refractive-index dispersion of bulk GaAs (the dashed-dotted curve) and the resultant double-peaked SHG coefficient (the dashed curve). The transitions in the asymmetric well are depicted in the inset. A confinement factor of $\sim 1 / 30$ in the quantum wells is needed for phase matching for $\tilde{\omega}=40$ and $\Delta \tilde{\omega}=3$.

Having calculated the potential of ISBT's designed for phase matching, we now consider the effect of the refractive-index contributions accompanying ISBT's designed for resonant enhancement of SHG. ISBT's in asymmetric three-level quantum wells have recently ${ }^{14,15}$ been used for near-resonant enhancement of SHG, and conversion efficiencies of $\sim 0.001$ at extremely short propagation distances have been reported. The assumption made in most of the reported analysis is that, since the propagation distances are much shorter than the bulk's coherence length, phase matching is unnecessary. This assumption neglects the index changes accompanying the ISBT's that could be tailored to offset the bulk's dispersion on one hand but could, on the other hand, significantly increase the phase mismatch. The magnitude of this ISBT-induced dispersion is easily estimated from the simple case of equal dipole elements and opposite detuning $\left[\mu_{12}=\mu_{13}\right.$ and $\left(\omega-\omega_{12}\right)=\left(\omega_{13}-2 \omega\right)$; see the inset of Fig. 3] for which

$$
\Delta n_{\mathrm{ISBT}}=n(\omega)-n(2 \omega) \cong \frac{1}{n} \chi_{\mathrm{res}} \frac{\Delta \tilde{\omega}}{\Delta \tilde{\omega}^{2}+1} .
$$

The accumulated phase difference at a distance of the inverse of the absorption coefficient is therefore $2 \Delta \tilde{\omega}$. Thus, when the normalized detuning $(\Delta \tilde{\omega})$ is not significantly smaller than $\pi / 2$, the ISBT phase mismatch may limit the maximal conversion efficiency, even for propagation distances shorter than the bulk's coherence length. In Fig. 3 we give an example of the ISBT's of a three-level quantum well, both enhancing SHG and offsetting the bulk's dispersion. The applicability of such a technique is limited by its high sensitivity to well parameters and by the saturation of near-resonant ISBT's, ${ }^{7,15}$ but the phase mismatch that may be caused by the ISBT's dispersion must be considered in any attempt to increase significantly the resonantly enhanced conversion efficiencies. ${ }^{14,15}$ Only on-resonant, or equally detuned, transitions of equal strength will not include such a phase mismatch.

In summary, we have considered the implications of the ISBT's index contributions to SHG and suggested a novel phase-matching technique. This technique replaces the coherence length limitations on SHG conversion efficiencies by absorption limitations - with a potential gain proportional to the transition's frequency-to-linewidth ratio squared. This technique may be applied to any material in which a desired wavelength transition with a sufficient refractive-index correction can be introduced. With current technology, the use of ISBT phase matching may lead to an improvement of almost 2 orders of magnitude in the bulk SHG of $5-\mu \mathrm{m}$ radiation in $\mathrm{GaAs}$, with further improvement dependent on the availability of narrower linewidth transitions.

This research was conducted with the support of the Advanced Research Projects Agency.

*Present address, Department of Electrical Engineering, Advanced Center for Photonics and Optoelectronic Materials and Princeton Material Institute, Princeton University, Princeton, New Jersey 08544.

\section{References}

1. J. A. Armstrong, N. Bloembergen, J. Ducuing, and P. S. Pershan, Phys. Rev. 127, 1918 (1962).

2. S. Somekh and A. Yariv, Appl. Phys. Lett. 21, 140 (1972).

3. G. Almogy, A. Shakouri, and A. Yariv, Appl. Phys. Lett. 63, 2720 (1993).

4. E. Dupont, D. Delacourt, and M. Papuchon, IEEE J. Quantum Electron. 29, 2313 (1993).

5. F. H. Julien, P. Vagos, J.-M. Lourtioz, and D. D. Yang, Appl. Phys. Lett. 59, 2645 (1991).

6. A possible exception is suggested by M. O. Scully, T. W. Hänsch, M. Fleischhauer, C. H. Keitel, and S. Y. Zhu, in Physics and Probability, W. T. Grandy, Jr., and P. W. Millonni, eds. (Cambridge U. Press, Cambridge, 1993), p. 73.

7. T. A. DeTemple, L. A. Bahler, and J. Osmundsen, Phys. Rev. A 24, 1950 (1981).

8. A. Yariv, Quantum Electronics (Wiley, New York, 1988).

9. E. D. Palik, ed., Handbook of Optical Constants of Solids (Academic, San Diego, Calif., 1991), Vol. 2, p. 534.

10. F. H. Julien, J. M. Lourtioz, N. Herschkorn, D. Delacourt, J. P. Pocholle, M. Papuchon, R. Planel, and G. Leroux, Appl. Phys. Lett. 53, 116 (1988).

11. R. J. Pressley, ed., Handbook of Lasers (CRC, Cleveland, Ohio, 1971), p. 504 (the crystal growth direction must correspond to a bulk SHG coefficient).

12. D. F. G. Gallagher, IEEE J. Quantum Electron. 28, 1785 (1992).

13. M. Wegner, T. Y. Chang, I. Bar-Joseph, J. M. Kuo, and D. S. Chemla, Appl. Phys. Lett. 55, 583 (1989).

14. M. M. Fejer, S. J. B. Yoo, R. L. Byer, A. Harwit, and J. S. Harris, Phys. Rev. Lett. 62, 1041 (1989).

15. P. Boucaud, F. H. Julien, D. D. Yang, J. M. Lourtioz, E. Rosencher, and P. Bois, Opt. Lett. 16, 199 (1991). 